\title{
Laboratory-based 3D X-ray microscopy of unirradiated U-10Zr fuel
}

Nikolaus Cordes ${ }^{1}$, Laura Sudderth ${ }^{2}$, Moiz Butt $^{3}$, James Jewell ${ }^{2}$, Mitchell Meyer ${ }^{2}$ and Sean McDeavitt ${ }^{3}$

${ }^{1}$ Idaho National Laboratory, Idaho Falls, Idaho, United States, ${ }^{2}$ Idaho National Laboratory, United States,

${ }^{3}$ Texas A\&M University, United States

Three dimensional (3D) X-ray microscopy, also referred to as X-ray computed tomography, is a data-rich characterization technique that can provide surface and subsurface spatial information in a non-destructive manner. ${ }^{1-3}$ However, 3D X-ray imaging of nuclear fuel is a challenging endeavor. ${ }^{4} \mathrm{X}$-ray attenuation of high-Z materials, such as uranium, can be significant. This limits the practical maximum X-ray path length (i.e., sample size) to a few millimeters at most. This physical limitation impedes the nondestructive microstructural imaging of real-world samples, such as fuel rods. However, scaling nuclear fuel samples to the appropriate size allows for X-ray path lengths that are suitable for high resolution, laboratory-based 3D X-ray microscopy.

Another challenge of 3D X-ray imaging highly attenuating samples is the presence of cupping artifacts in the resultant 3D tomogram, which is caused by X-ray beam hardening. This occurs from the use of a polychromatic X-ray beam in which a significant portion of the emitted low X-ray energy photons are attenuated by the sample. High energy X-ray photons pass through the sample to the detector, thus providing a radiographic image. The reconstructed tomogram, however, may present this cupping artifact and prevent facile image segmentation based on grayscale intensity. Beam hardening can be reduced during image acquisition by using a physical X-ray filter to adjust the X-ray energy range or the cupping artifact can be corrected during either reconstruction or post-reconstruction using digital image processing. The combination of these methods is typically sufficient for a large number of sample types, but can be ineffective for samples with very low X-ray transmissions.

This presentation will give an overview of laboratory-based 3D X-ray microscopy of appropriately sized, unirradiated U-10Zr fuel cylinders as part of a Nuclear Science User Facility-funded collaborative project to validate Idaho National Laboratory's MARMOT mesoscale fuel performance code. The challenges of imaging these samples, and their solutions, will be addressed. These include the development of a novel, GPU-accelerated post-reconstruction cupping artifact correction method and the implementation of pattern recognition-based segmentation methods ${ }^{5,6}$ using open-source image processing software ${ }^{7,8}$.

Funding Acknowledgment:

The MARMOT Validation Project is a joint Nuclear Science User Facilities research collaboration between Texas A\&M University and Idaho National Laboratory. This work was partially supported by the U.S. Department of Energy, Office of Nuclear Energy under DOE Idaho Operations Office Contract DE-AC07- 051D14517 as part of a Nuclear Science User Facilities project.

References

1. Maire, E.; Withers, P. J., Quantitative X-ray tomography. International Materials Reviews 2014, 59 (1), 1-43.

2. Stock, S. R., Recent advances in X-ray microtomography applied to materials. International Materials Reviews 2008, 53 (3), 129-181. 
3. Dunsmuir, J. H.; Bennett, S.; Fareria, L.; Mingino, A.; Sansone, M., X-ray microtomographic imaging and analysis for basic research. Powder Diffraction 2006, 21 (2), 125-131.

4. Cordes, N.; Kane, J.; Craft, A., 3D X-ray Microscopy of Nuclear Energy Materials. Microscopy and Microanalysis 2020, 26 (S2), 872-872.

5. Kanungo, T.; Mount, D. M.; Netanyahu, N. S.; Piatko, C. D.; Silverman, R.; Wu, A. Y., An efficient k-means clustering algorithm: analysis and implementation. IEEE Transactions on Pattern Analysis and Machine Intelligence 2002, 24 (7), 881-892.

6. Bezdek, J. C.; Ehrlich, R.; Full, W., FCM: The fuzzy c-means clustering algorithm. Computers \& Geosciences 1984, 10 (2), 191-203.

7. Schindelin, J.; Arganda-Carreras, I.; Frise, E.; Kaynig, V.; Longair, M.; Pietzsch, T.; Preibisch, S.; Rueden, C.; Saalfeld, S.; Schmid, B.; Tinevez, J.-Y.; White, D. J.; Hartenstein, V.; Eliceiri, K.; Tomancak, P.; Cardona, A., Fiji: an open-source platform for biological-image analysis. Nature Methods 2012, 9 (7), 676-682.

8. Haase, R.; Royer, L. A.; Steinbach, P.; Schmidt, D.; Dibrov, A.; Schmidt, U.; Weigert, M.; Maghelli, N.; Tomancak, P.; Jug, F.; Myers, E. W., CLIJ: GPU-accelerated image processing for everyone. Nature Methods 2020, 17 (1), 5-6. 